\title{
SOME PROPERTIES OF BIVATIATE FIBONACCI AND LUCAS QUATERNION POLYNOMIALS
}

\author{
Arzu Özkoç Öztürk and Faruk Kaplan
}

\begin{abstract}
(C) 2020 by University of Niš, Serbia | Creative Commons License: CC BY-NC-ND
\end{abstract}
Abstract. In this work, we introduce bivariate Fibonacci quaternion polynomials and bivariate Lucas quaternion polynomials. We present generating function, Binet formula, matrix representation, binomial formulas and some basic identities for the bivariate Fibonacci and Lucas quaternion polynomial sequences. Moreover we give various kinds of sums for these quaternion polynomials.

Keywords: Bivariate Fibonacci quaternion polynomials, Bivariate Lucas quaternion polynomials, Generating function, Binet formula.

\section{Introduction}

In mathematics, Fibonacci and Lucas or other special numbers are investigation topic of great interest. Classical Fibonacci sequence $\left\{F_{n}\right\}_{n \in \mathbb{N}}$ is defined by a recurrence identity;

$$
F_{n}=\left\{\begin{array}{ccc}
0 & \text { if } & n=0 \\
1 & \text { if } & n=1 \\
F_{n-1}+F_{n-2} & \text { if } & n \geq 2
\end{array}\right.
$$

The Lucas sequence $\left\{L_{n}\right\}_{n \in \mathbb{N}}$ is defined by some recurrence identity with different starting values;

$$
L_{n}=\left\{\begin{array}{ccc}
2 & \text { if } & n=0 \\
1 & \text { if } & n=1 \\
L_{n-1}+L_{n-2} & \text { if } & n \geq 2
\end{array}\right.
$$

Received September 21, 2018; accepted Octover 10, 2018

2010 Mathematics Subject Classification. Primary 11B39 ; Secondary 11R52 
Let $p(x)$ and $q(x)$ be polynomials with real coefficients of the $(p, q)$-Fibonacci polynomials are defined by the recurrence relation

$$
F_{p, q, n+1}=p(x) F_{p, q, n}+q(x) F_{p, q, n-1}
$$

with the initial conditions $F_{p, q, 0}=0, F_{p, q, 1}=1$. Also for the $p(x)$ and $q(x)$ polynomials with real coefficients the $(p, q)$-Lucas polynomials are defined by the recurrence relation

$$
L_{p, q, n+1}=p(x) L_{p, q, n}+q(x) L_{p, q, n-1}
$$

with initial conditions $L_{p, q, 0}=2, L_{p, q, 1}=p(x)$.

Definition 1.1. [1] For $n \geq 2$, bivariate Fibonacci polynomials are defined as recurrence relation

$$
F_{n}(x, y)=x F_{n-1}(x, y)+y F_{n-2}(x, y)
$$

We can compute the first few bivariate Fibonacci polynomials as follow $F_{0}(x, y)=$ $0, \quad F_{1}(x, y)=1, \quad F_{2}(x, y)=x, \quad F_{3}(x, y)=x^{2}+y, \quad F_{4}(x, y)=x^{3}+2 x y$. Characteristic equation of relation (1.1) is

$$
h^{2}-x h-y=0
$$

and so the roots of $(1.2)$ are $\alpha=\alpha(x, y)=\frac{x+\sqrt{x^{2}+4 y}}{2}$ and $\beta=\beta(x, y)=$ $\frac{x-\sqrt{x^{2}+4 y}}{2}$. Also it has Binet's formula $F_{n}(x, y)=\frac{\alpha^{n}-\beta^{n}}{\alpha-\beta}$ for $n \geq 0$.

Definition 1.2. [1] For $n \geq 2$, bivariate Lucas polynomials are defined as recurrence relation

$$
L_{n}(x, y)=x L_{n-1}(x, y)+y L_{n-2}(x, y)
$$

with initial conditionals $L_{0}(x, y)=2$ and $L_{1}(x, y)=1$.

Likely, let compute the first few terms of Lucas polynomials $L_{0}(x, y)=2$, $L_{1}(x, y)=1, L_{2}(x, y)=x+2 y, L_{3}(x, y)=x^{2}+2 x y+y, L_{4}(x, y)=x^{3}+2 x^{2} y+$ $2 x y+2 y^{2}$. Also it has Binet's formula $L_{n}(x, y)=\alpha^{n}+\beta^{n}$ for $n \geq 0$.

Some authors considered special sequence polynomials for example generalized Fibonacci and Lucas polynomials in [7] and also bivariate Fibonacci and Lucas like polynomials in [6].

Normed division algebra, nowadays which is so important topic consists of the real numbers $\mathbb{R}$, complex numbers $\mathbb{C}$, quaternions $\mathbf{H}$ and octonions $\mathbf{O}$. Prime facie, directly we can not extend sundry results on real and complex numbers to quaternions due to quaternions are noncommutative normed division algebra over the real 
numbers, even it looks like things are going to be done with quaternions $\mathbf{H}$ [3]. For $a_{0}, a_{1}, a_{2}, a_{3} \in \mathbb{R}$, a quaternion is defined by

$$
e=a_{0} e_{0}+a_{1} e_{1}+a_{2} e_{2}+a_{3} e_{3}
$$

where $e_{0}=1, e_{1}, e_{2}$, and $e_{3}$ are unit vectors which verifies the following rules

$$
\left(e_{1}\right)^{2}=\left(e_{2}\right)^{2}=\left(e_{3}\right)^{2}=e_{1} e_{2} e_{3}=-1
$$

From equation (1.3), we get

$$
e_{1} e_{2}=-e_{2} e_{1}=e_{3}, \quad e_{2} e_{3}=-e_{3} e_{2}=e_{1}, \quad e_{1} e_{3}=-e_{3} e_{1}=e_{2}
$$

Some new quaternion and octonion polynomials are studied in $[2,4,5,8,9]$.

\section{Bivariate Fibonacci and Lucas quaternion polynomials}

Now, we define new quaternion polynomials which are called bivariate Fibonacci quaternion polynomials $(Q B F)$ and bivariate Lucas quaternion polynomials $(Q B L)$.

Definition 2.1. Bivariate Fibonacci quaternion polynomials $(Q B F)$ are defined as the recurrence relation

$$
\begin{aligned}
Q B F_{n}(x, y) & =\sum_{k=0}^{3} F_{n+k}(x, y) e_{k} \\
& =F_{n}(x, y) e_{0}+F_{n+1}(x, y) e_{1}+F_{n+2}(x, y) e_{2}+F_{n+3}(x, y) e_{3}
\end{aligned}
$$

where $F_{n+k}(x, y)$ is the $n-t h$ bivariate Fibonacci polynomial with the initial conditions $Q B F_{0}(x, y)=e_{1}+x e_{2}+\left(x^{2}+y\right) e_{3}$ and $Q B F_{1}(x, y)=e_{0}+x e_{1}+\left(x^{2}+\right.$ $y) e_{2}+\left(x^{3}+2 x y\right) e_{3}$.

Furthermore,

$$
\begin{aligned}
Q B F_{n+1}(x, y) & =\sum_{k=0}^{3} F_{n+1+k}(x, y) e_{k} \\
& =x \sum_{k=0}^{3} F_{n+k}(x, y) e_{k}+y \sum_{k=0}^{3} F_{n+k-1}(x, y) e_{k}
\end{aligned}
$$

So we get recurrence relation as follow

$$
Q B F_{n+1}(x, y)=x Q B F_{n}(x, y)+y Q B F_{n-1}(x, y)
$$


Similarly, bivariate Lucas quaternion polynomials $Q B L$ are defined as the recurrence relation

$$
\begin{aligned}
Q B L_{n}(x, y) & =\sum_{k=0}^{3} L_{n+k}(x, y) e_{k} \\
& =L_{n}(x, y) e_{0}+L_{n+1}(x, y) e_{1}+L_{n+2}(x, y) e_{2}+L_{n+3}(x, y) e_{3}
\end{aligned}
$$

where $L_{n+k}(x, y)$ is the $n-t h$ bivariate Lucas polynomial and with the initial conditions $Q B L_{0}(x, y)=2 e_{0}+e_{1}+(x+2 y) e_{2}+\left(x^{2}+2 x y+y\right) e_{3}$ and $Q B L_{1}(x, y)=$ $e_{0}+(x+y) e_{1}+\left(x^{2}+2 x y+y\right) e_{2}+\left(x^{3}+2 x^{2} y+2 x y+2 y^{2}\right) e_{3}$. Moreover, recurrence relation is

$\quad Q B L_{n+1}(x, y)=x Q B L_{n}(x, y)+y Q B L_{n-1}(x, y)$.

Let $\alpha(x, y)=\frac{x+\sqrt{x^{2}+4 y}}{2}$ and $\beta(x, y)=\frac{x-\sqrt{x^{2}+4 y}}{2}$ denote the roots of the characteristic equation such that $\sqrt{x^{2}+4 y}=\Delta$,

$$
t^{2}-x t-y t=0
$$

on the recurrence relation of (2.2) and (2.4).

From now on, for convenience of representation, we adopt the following notation

$$
\alpha(x, y)=\alpha, \beta(x, y)=\beta, \Delta=\sqrt{x^{2}+4 y} .
$$

Equations that can be obtained with these roots are as follow

$$
\begin{aligned}
\alpha+\beta & =x \\
\alpha-\beta & =\Delta \\
\alpha \beta & =-y \\
\frac{\alpha}{\beta} & =-\frac{\alpha^{2}}{y} \\
\frac{\beta}{\alpha} & =-\frac{\beta^{2}}{y} .
\end{aligned}
$$

We continue with the generating function results.

Theorem 2.1. The generating functions for $Q B F$ and $Q B L$ polynomials are respectively

$$
\sum_{n=0}^{\infty} Q B F_{n}(x, y) t^{n}=\frac{Q B F_{0}(x, y)+\left[Q B F_{1}(x, y)-x Q B F_{0}(x, y)\right] t}{1-x t-y t^{2}}
$$


and

$$
\sum_{n=0}^{\infty} Q B L_{n}(x, y) t^{n}=\frac{Q B L_{0}(x, y)+\left[Q B L_{1}(x, y)-x Q B L_{0}(x, y)\right] t}{1-x t-y t^{2}}
$$

Proof. To compute the generating function of $Q B F$ polynomials

$$
\begin{aligned}
& \sum_{n=0}^{\infty} Q B F_{n}(x, y) t^{n} \\
= & Q B F_{0}(x, y)+Q B F_{1}(x, y) t+Q B F_{2}(x, y) t^{2}+\cdots+Q B F_{n}(x, y) t^{n}+\cdots
\end{aligned}
$$

then using the equations of $-x t\left(\sum_{n=0}^{\infty} Q B F_{n}(x, y) t^{n}\right)$ and $-y t^{2}\left(\sum_{n=0}^{\infty} Q B F_{n}(x, y) t^{n}\right)$

$$
\begin{aligned}
& \sum_{n=0}^{\infty} Q B F_{n}(x, y) t^{n}+(-x t) \sum_{n=0}^{\infty} Q B F_{n}(x, y) t^{n}+\left(-y t^{2}\right) \sum_{n=0}^{\infty} Q B F_{n}(x, y) t^{n} \\
= & Q B F_{0}(x, y)+\left[Q B F_{1}(x, y)-x Q B F_{0}(x, y)\right] t \\
& +\left[Q B F_{2}(x, y)-x Q B F_{1}(x, y)-y Q B F_{0}(x, y)\right] t^{2} \\
& +\cdots+\left[Q B F_{n}(x, y)-x Q B F_{n-1}(x, y)-y Q B F_{n-2}(x, y)\right] t^{n}+\cdots
\end{aligned}
$$

Consequently,

$\sum_{n=0}^{\infty} Q B F_{n}(x, y) t^{n}\left(1-x t-y t^{2}\right)=Q B F_{0}(x, y)+\left(Q B F_{1}(x, y)-x Q B F_{0}(x, y)\right) t$

is valid. Similar proof can be done for $Q B L$ polynomials.

Now we can give the following theorems.

Lemma 2.1. If we rearrange the Theorem 2.1, we have the generating functions as follows

$$
\sum_{n=0}^{\infty} Q B F_{n}(x, y) t^{n}=\frac{\frac{Q B F_{1}(x, y)-\beta Q B F_{0}(x, y)}{1-\alpha t}-\frac{Q B F_{1}(x, y)-\alpha(x, y) Q B F_{0}(x, y)}{1-\beta t}}{\alpha-\beta}
$$

and

$$
\sum_{n=0}^{\infty} Q B L_{n}(x, y) t^{n}=\frac{\frac{Q B L_{1}(x, y)-\beta Q B L_{0}(x, y)}{1-\alpha t}-\frac{Q B L_{1}(x, y)-\alpha(x, y) Q B L_{0}(x, y)}{1-\beta t}}{\alpha-\beta} .
$$


Proof. If we use Theorem 2.1 and recurrence relation (2.2), then we have

$$
\begin{aligned}
& \sum_{n=0}^{\infty} Q B F_{n}(x, y) t^{n} \\
& =\left(\frac{Q B F_{0}(x, y)+\left(Q B F_{1}(x, y)-(\alpha+\beta) Q B F_{0}(x, y)\right) t}{(1-\alpha t)(1-\beta t)}\right) \\
& \times\left(\frac{\alpha-\beta}{\alpha-\beta}\right) \\
& =\frac{\left\{\begin{array}{c}
Q B F_{1}(x, y)(1-\beta t)+\beta Q B F_{0}(x, y)(-1+\beta t) \\
+Q B F_{1}(x, y)(-1+\alpha t)+\alpha Q B F_{0}(x, y)(1-\alpha t)
\end{array}\right\}}{(1-\alpha t)(1-\beta t)(\alpha-\beta)} \\
& =\frac{\frac{Q B F_{1}(x, y)-\beta Q B F_{0}(x, y)}{1-\alpha t}-\frac{Q B F_{1}(x, y)-\alpha(x, y) Q B F_{0}(x, y)}{1-\beta t}}{\alpha-\beta} .
\end{aligned}
$$

Hence the proof is completed. The other $Q B L$ polynomials can be proved similarly.

Lemma 2.2. For $k \geq 0$, let bivariate Fibonacci and Lucas polynomials are $F_{n}(x, y)$ and $L_{n}(x, y)$. We have

$$
\begin{aligned}
\text { (i) } F_{k+1}(x, y)-\alpha F_{k}(x, y) & =\beta^{k} \\
\text { (ii) } F_{k+1}(x, y)-\beta F_{k}(x, y) & =\alpha^{k} \\
\text { (iii) } \frac{\alpha L_{k}(x, y)-L_{k+1}(x, y)}{\alpha-\beta} & =\beta^{k} \\
\text { (iv) } \frac{L_{k+1}(x, y)-\beta L_{k}(x, y)}{\alpha-\beta} & =\alpha^{k} .
\end{aligned}
$$

Proof. (i) We can prove it by induction method. Let $k=1$, then $F_{2}(x, y)-$ $\alpha F_{1}(x, y)=\beta$.

Now let us assume that the equation is $F_{n}(x, y)-\alpha F_{n-1}(x, y)=\beta^{n-1}$, for $k=n-1$. For $k=n$ it becomes,

$$
\begin{aligned}
\beta^{n} & =\beta^{n-1} \beta \\
& =\left(\left(F_{n}(x, y)-\alpha F_{n-1}(x, y)\right) \beta\right. \\
& =\beta F_{n}(x, y)-\alpha \beta F_{n-1}(x, y) \\
& =(\alpha+\beta-\alpha) F_{n}(x, y)-\alpha F_{n}(x, y)-\alpha \beta F_{n}(x, y) \\
& =x F_{n}(x, y)+y F_{n}(x, y)-\alpha F_{n}(x, y) \\
& =F_{n-1}(x, y)-\alpha F_{n}(x, y) .
\end{aligned}
$$

so this completes the proof. $(i i),(i i i)$ and $(i v)$ can be done similarly. 
Now we want to derive the Binet formulas for $Q B F$ and $Q B L$ polynomials. To get this we can give the following theorem.

Theorem 2.2. The Binet formulas of $Q B F$ and $Q B L$ polynomials are given as

$$
\begin{aligned}
Q B F_{n}(x, y) & =\frac{\alpha^{*} \alpha^{n}-\beta^{*} \beta^{n}}{\alpha-\beta} \\
Q B L_{n}(x, y) & =\alpha^{*} \alpha^{n}+\beta^{*} \beta^{n} \\
\text { for } n \geq 0, \text { where } \alpha^{*}=\sum_{k=0}^{3} \alpha^{k} e_{k} \text { and } \beta^{*} & =\sum_{k=0}^{3} \beta^{k} e_{k} .
\end{aligned}
$$

Proof. Recall that generating function is

$$
\sum_{n=0}^{\infty} Q B F_{n}(x, y) t^{n}=\frac{Q B F_{0}(x, y)+\left(Q B F_{1}(x, y)-x Q B F_{0}(x, y)\right) t}{1-x t-y t^{2}}
$$

So using the Lemma 2.1 and Lemma 2.2, we have

$$
\begin{aligned}
& \sum_{n=0}^{\infty} Q B F_{n}(x, y) t^{n} \\
= & \sum_{k=0}^{\infty}\left(F_{k+1}-\beta F_{k+1}\right) e_{k} \sum_{n=0}^{\infty} \alpha^{n} t^{n}-\sum_{k=0}^{\infty}\left(F_{k+1}-\alpha F_{k+1}\right) e_{k} \sum_{n=0}^{\infty} \beta^{n} t^{n} .
\end{aligned}
$$

So we get,

$$
\sum_{n=0}^{\infty}\left(\frac{\alpha^{*} \alpha^{n}-\beta^{*} \beta^{n}}{\alpha-\beta}\right) t^{n}
$$

this is valid. Binet formula for the other $Q B L$ polynomial can be done similarly.

We derive generating functions for the $(m k+s)-t h$ order of $Q B F$ and $Q B L$ polynomials.

Theorem 2.3. For all $n \in \mathbb{N}$ and $m, s \in \mathbb{Z}$, we have

$$
\sum_{k=0}^{\infty} Q B F_{m k+s}(x, y) x^{k}=\frac{Q B F_{s}(x, y)-(-y)^{m} Q B F_{s-m}(x, y) x}{(-y)^{m}-L_{m}(x, y)+1}
$$

and

$$
\sum_{k=0}^{\infty} Q B L_{m k+s}(x, y) x^{k}=\frac{Q B L_{s}(x, y)-(-y)^{m} Q B L_{s-m}(x, y) x}{(-y)^{m}-L_{m}(x, y)+1}
$$


Proof. Using Binet formula and equation (2.5), we have

$$
\begin{aligned}
& \sum_{k=0}^{\infty} Q B F_{m k+s}(x, y) x^{k} \\
& =\sum_{k=0}^{\infty} \frac{\alpha^{*} \alpha^{m k+s}-\beta^{*} \beta^{m k+s}}{\alpha-\beta} x^{k} \\
& =\frac{\alpha^{*} \alpha^{s}}{\alpha-\beta} \sum_{k=0}^{\infty} \alpha^{m k} x^{k}-\frac{\beta^{*} \beta^{s}}{\alpha-\beta} \sum_{k=0}^{\infty} \beta^{m k} x^{k} \\
& =\frac{\alpha^{*} \alpha^{s}}{\alpha-\beta}\left(\frac{1}{1-\alpha^{m} x}\right)-\frac{\beta^{*} \beta^{s}}{\alpha-\beta}\left(\frac{1}{1-\beta^{m} x}\right) \\
& =\frac{\frac{\alpha^{*} \alpha^{s}-\beta^{*} \beta^{s}}{\alpha-\beta}-(\alpha \beta)^{m}\left(\frac{\alpha^{*} \alpha^{s-m}-\beta^{*} \beta^{s-m}}{\alpha-\beta}\right) x}{1-\left(\alpha^{m}+\beta^{m}\right) x+(\alpha \beta)^{m} x^{2}}
\end{aligned}
$$

this is valid. The other result can be done similarly.

We formulate the sum of the first $n$ terms of these sequences of $Q B F$ and $Q B L$ polynomials.

Theorem 2.4. The sum of the first $n$-terms of the quaternion sequences $Q B F_{n}(x, y)$ and $Q B L_{n}(x, y)$ is given by

$$
\sum_{k=0}^{n} Q B F_{k}(x, y)=\frac{\left\{\begin{array}{c}
-y Q B F_{n}(x, y)-Q B F_{n+1}(x, y) \\
+Q B F_{0}(x, y)-\frac{\alpha^{*} \beta-\beta^{*} \alpha}{\alpha-\beta}
\end{array}\right\}}{(\alpha-1)(\beta-1)}
$$

and

$$
\sum_{k=0}^{n} Q B L_{k}(x, y)=\frac{\left\{\begin{array}{c}
-y Q B L_{n}(x, y)-Q B L_{n+1}(x, y) \\
+Q B L_{0}(x, y)+\alpha^{*} \beta+\beta^{*} \alpha
\end{array}\right\}}{(\alpha-1)(\beta-1)}
$$

Proof. Using Binet formula and equation (2.5), we get

$$
\begin{aligned}
& \sum_{k=0}^{n} Q B F_{k}(x, y) \\
= & \sum_{k=0}^{n} \frac{\alpha^{*} \alpha^{k}-\beta^{*} \beta^{k}}{\alpha-\beta} \\
= & \frac{1}{\alpha-\beta}\left\{\alpha^{*} \sum_{k=0}^{n} \alpha^{k}-\beta^{*} \sum_{k=0}^{n} \beta^{k}\right\}
\end{aligned}
$$




$$
\begin{aligned}
& =\frac{1}{\alpha-\beta}\left\{\alpha^{*}\left(\frac{\alpha^{n+1}-1}{\alpha-1}\right)-\beta^{*}\left(\frac{\beta^{n+1}-1}{\beta-1}\right)\right\} \\
& =\frac{1}{(\alpha-1)(\beta-1)}\left\{\begin{array}{c}
\frac{\alpha \beta\left(\alpha^{*} \alpha^{n}-\beta^{*} \beta^{n}\right)}{\alpha-\beta}-\frac{\alpha^{*} \alpha^{n+1}-\beta^{*} \beta^{n+1}}{\alpha-\beta} \\
+\frac{\alpha^{*}-\beta^{*}}{\alpha-\beta}-\frac{\alpha^{*} \beta-\alpha \beta^{*}}{\alpha-\beta}
\end{array}\right\} .
\end{aligned}
$$

The other case can be done similarly.

We derive summation formulas for the $(m k+s)-t h$ order of $Q B F$ and $Q B L$ polynomials.

Theorem 2.5. For all $n \in \mathbb{N}$ and $m, s \in \mathbb{Z}$, we have

$$
\sum_{k=0}^{n} Q B F_{m k+s}(x, y)=\frac{\left\{\begin{array}{c}
(-y)^{m}\left(Q B F_{m n+s}(x, y)-Q B F_{s-m}(x, y)\right) \\
-Q B F_{m n+m+s}(x, y)+Q B F_{s}(x, y)
\end{array}\right\}}{(-y)^{m}-F_{m}(x, y)+1}
$$

and

$$
\sum_{k=0}^{n} Q B L_{m k+s}(x, y)=\frac{\left\{\begin{array}{c}
(-y)^{m}\left(Q B L_{m n+s}(x, y)-Q B L_{s-m}(x, y)\right) \\
-Q B L_{m n+m+s}(x, y)+Q B L_{s}(x, y)
\end{array}\right\}}{(-y)^{m}-L_{m}(x, y)+1}
$$

Proof. Using Binet formula, equation (2.5), we have

$$
\begin{aligned}
& \sum_{k=0}^{n} Q B F_{m k+s}(x, y) \\
= & \sum_{k=0}^{n} \frac{\alpha^{*} \alpha^{m k+s}-\beta^{*} \beta^{m k+s}}{\alpha-\beta} \\
= & \frac{\alpha^{*} \alpha^{s}}{\alpha-\beta}\left(\frac{\alpha^{m n+m}-1}{\alpha^{m}-1}\right)-\frac{\alpha^{*} \alpha^{s}}{\alpha-\beta}\left(\frac{\alpha^{m n+m}-1}{\alpha^{m}-1}\right) \\
= & \frac{\left\{\begin{array}{c}
\alpha^{*}\left(\alpha^{m n+s} \alpha^{m} \beta^{m}-\alpha^{m n+m+s}-\alpha^{s} \beta^{m}+\alpha^{s}\right) \\
-\beta^{*}\left(\beta^{m n+s} \alpha^{m} \beta^{m}-\beta^{m n+m+s}-\alpha^{m} \beta^{s}+\beta^{s}\right)
\end{array}\right\}}{(\alpha-\beta)\left(\alpha^{m} \beta^{m}-\alpha^{m}-\beta^{m}+1\right)} \\
= & \frac{(\alpha \beta)^{m}\left(\alpha^{*} \alpha^{m n+s}-\beta^{*} \alpha^{m n+s}\right)-\left(\alpha^{*} \alpha^{m n+m+s}-\beta^{*} \beta^{m n+m+s}\right)}{(\alpha-\beta)\left(\alpha^{m} \beta^{m}-\alpha^{m}-\beta^{m}+1\right)} \\
& +\frac{-(\alpha \beta)^{m}\left(\alpha^{*} \alpha^{s-m}-\beta^{*} \alpha^{s-m}\right)-\left(\alpha^{*} \alpha^{m n+m+s}-\beta^{*} \beta^{m n+m+s}\right)}{(\alpha-\beta)\left(\alpha^{m} \beta^{m}-\alpha^{m}-\beta^{m}+1\right)} .
\end{aligned}
$$

Other case can be done similarly. 
Now, some new results for binomial summation of these sequences are derived by using their Binet forms.

Theorem 2.6. Let $n$ be a non-negative integer. Then we have the following binomial sum formulas for odd and even terms,

$$
\begin{aligned}
\text { (i) } \sum_{k=0}^{n}\left(\begin{array}{l}
n \\
k
\end{array}\right) y^{n-k} x^{k} Q B F_{k}(x, y) & =Q B F_{2 n}(x, y) \\
\text { (ii) } \sum_{k=0}^{n}\left(\begin{array}{l}
n \\
k
\end{array}\right) y^{n-k} x^{k} Q B F_{k}(x, y) & =Q B F_{2 n+1}(x, y) \\
\text { (iii) } \sum_{k=0}^{n}\left(\begin{array}{l}
n \\
k
\end{array}\right) y^{n-k} x^{k} Q B L_{k}(x, y) & =Q B L_{2 n}(x, y) \\
\text { (iv) } \sum_{k=0}^{n}\left(\begin{array}{l}
n \\
k
\end{array}\right) y^{n-k} x^{k} Q B L_{k}(x, y) & =Q B L_{2 n+1}(x, y) .
\end{aligned}
$$

Proof. (i) Let $P=\sum_{k=0}^{n}\left(\begin{array}{l}n \\ k\end{array}\right) y^{n-k} x^{k} Q B F_{k}(x, y)$. From Binet formula, we change the right-hand side of $P$ into:

$$
P=\sum_{k=0}^{n}\left(\begin{array}{l}
n \\
k
\end{array}\right) y^{n-k} x^{k}\left(\frac{\alpha^{*} \alpha^{k}-\beta^{*} \beta^{k}}{\alpha-\beta}\right) .
$$

Elementary calculations implies that

$$
P=\frac{\alpha^{*}(y+x \alpha)^{n}-\beta^{*}(y+x \beta)^{n}}{\alpha-\beta} .
$$

From equation (2.5), we get

$$
\frac{\alpha^{*} \alpha^{2 n}-\beta^{*} \beta^{2 n}}{\alpha-\beta}=Q B F_{2 n}(x, y) .
$$

The other cases $(i i),(i i i)$ and $(i v)$ can be done similarly.

Now we can also formulate the Catalan's identity, Cassini's identity and d'Ocagne's identity by using Binet formulas.

Theorem 2.7. (Catalan's Identity) For $n$ and $k$ non-negative integer such that $k \leq n$, we have

$$
\begin{aligned}
& Q B F_{n+k}(x, y) Q B F_{n-k}(x, y)-Q B F_{n}^{2}(x, y) \\
= & (-y)^{n-k} F_{n-k}(x, y)\left(\frac{\alpha^{*} \beta^{*} \beta^{k}-\beta^{*} \alpha^{*} \alpha^{k}}{(\alpha-\beta)}\right)
\end{aligned}
$$

and

$$
\begin{aligned}
& Q B L_{n+k}(x, y) Q B L_{n-k}(x, y)-Q B L_{n}^{2}(x, y) \\
= & (-y)^{n-k} F_{n-k}(x, y) \sqrt{\triangle}\left(\alpha^{*} \beta^{*} \beta^{k}-\beta^{*} \alpha^{*} \alpha^{k}\right) .
\end{aligned}
$$


Proof. Using Binet formula, we obtain

$$
\begin{aligned}
& Q B F_{n+k}(x, y) Q B F_{n-k}(x, y)-Q B F_{n}^{2}(x, y) \\
= & \left(\frac{\alpha^{*} \alpha^{n+k}-\beta^{*} \beta^{n+k}}{\alpha-\beta}\right)\left(\frac{\alpha^{*} \alpha^{n-k}-\beta^{*} \beta^{n-k}}{\alpha-\beta}\right)-\left(\frac{\alpha^{*} \alpha^{n}-\beta^{*} \beta^{n}}{\alpha-\beta}\right) \\
= & \frac{(\alpha \beta)^{n}}{(\alpha-\beta)^{2}}\left(\alpha^{*} \beta^{*}\left(\frac{\alpha^{k}-\beta^{k}}{\alpha-\beta}\right)+\beta^{*} \alpha^{*}\left(\frac{\beta^{k}-\alpha^{k}}{\alpha-\beta}\right)\right) \\
= & (\alpha \beta)^{n-k}\left(\frac{\alpha^{k}-\beta^{k}}{\alpha-\beta}\right)\left(\frac{\alpha^{*} \beta^{*} \beta^{k}-\beta^{*} \alpha^{*} \alpha^{k}}{\alpha-\beta}\right) .
\end{aligned}
$$

Theorem 2.8. For any natural number $n$, Cassini's identities for $Q B F$ and $Q B L$ polynomials are

$$
Q B F_{n+1}(x, y) Q B F_{n-1}(x, y)-Q B F_{n}^{2}(x, y)=(-y)^{n-1}\left(\frac{\alpha^{*} \beta^{*} \beta-\beta^{*} \alpha^{*} \alpha}{\alpha-\beta}\right)
$$

and

$$
Q B L_{n+1}(x, y) Q B L_{n-1}(x, y)-Q B L_{n}^{2}(x, y)=(-y)^{n-1} \sqrt{\triangle}\left(\alpha^{*} \beta^{*} \beta-\beta^{*} \alpha^{*} \alpha\right) .
$$

Proof. Let $k=1$ in Catalan's identity so the proof is completed for both of $Q B F$ and $Q B L$ polynomials.

Theorem 2.9. (d'Ocagne's Identity) Let $Q B F_{n}$ and $Q B L_{n}$ be $n$-th $Q B F$ and QBL polynomials. The d'Ocagne's identities are

$$
\begin{aligned}
& Q B F_{k}(x, y) Q B F_{n+1}(x, y)-Q B F_{k+1}(x, y) Q B F_{n}(x, y) \\
= & \frac{(-1)^{n} y^{n}}{\alpha-\beta}\left(\alpha^{*} \beta^{*} \alpha^{k-n}-\beta^{*} \alpha^{*} \beta^{k-n}\right)
\end{aligned}
$$

and

$$
\begin{aligned}
& Q B L_{k}(x, y) Q B L_{n+1}(x, y)-Q B L_{k+1}(x, y) Q B L_{n}(x, y) \\
= & (\alpha-\beta)\left(\beta^{*} \alpha^{*} \beta^{k} \alpha^{n}-\alpha^{*} \beta^{*} \alpha^{k} \beta^{n}\right) .
\end{aligned}
$$

Proof. From Binet formula to left -hand side, we get

$$
\begin{aligned}
& Q B F_{k}(x, y) Q B F_{n+1}(x, y)-Q B F_{k+1}(x, y) Q B F_{n}(x, y) \\
= & \left(\frac{\alpha^{*} \alpha^{k}-\beta^{*} \beta^{k}}{\alpha-\beta}\right)\left(\frac{\alpha^{*} \alpha^{n+1}-\beta^{*} \beta^{n+1}}{\alpha-\beta}\right)-\left(\frac{\alpha^{*} \alpha^{k+1}-\beta^{*} \beta^{k+1}}{\alpha-\beta}\right)\left(\frac{\alpha^{*} \alpha^{n}-\beta^{*} \beta^{n}}{\alpha-\beta}\right)
\end{aligned}
$$




$$
\begin{aligned}
& =\frac{1}{(\alpha-\beta)^{2}}\left\{\begin{array}{c}
\left(\alpha^{*}\right)^{2} \alpha^{n+k+1}-\beta^{*} \alpha^{*} \beta^{k} \alpha^{n+1}-\alpha^{*} \beta^{*} \alpha^{k} \beta^{n+1}+\left(\beta^{*}\right)^{2} \beta^{n+k+1} \\
-\left(\alpha^{*}\right)^{2} \alpha^{n+k+1}+\beta^{*} \alpha^{*} \beta^{k+1} \alpha^{n}+\alpha^{*} \beta^{*} \alpha^{k+1} \beta^{n}-\left(\beta^{*}\right)^{2} \beta^{n+k+1}
\end{array}\right\} \\
& =\frac{\beta^{*} \alpha^{*} \beta^{k} \alpha^{n}(\beta-\alpha)+\alpha^{*} \beta^{*} \alpha^{k} \beta^{n}(\alpha-\beta)}{(\alpha-\beta)^{2}} \\
& =\frac{(\alpha \beta)^{n}}{(\alpha-\beta)}\left(\alpha^{*} \beta^{*} \alpha^{k-n}-\beta^{*} \alpha^{*} \beta^{k-n}\right) .
\end{aligned}
$$

The other case can be done similarly.

The corresponding identities for $Q B F$ and $Q B L$ polynomials are contained in the next theorem.

Theorem 2.10. For $n \geq 0$, the following statements hold:

$$
y Q B F_{n}^{2}(x, y)+Q B F_{n+1}^{2}(x, y)=\frac{\left(\alpha^{*}\right)^{2} \alpha^{2 n+1}-\left(\beta^{*}\right)^{2} \beta^{2 n+1}}{\alpha-\beta}
$$

and

$$
y Q B L_{n}^{2}(x, y)+Q B L_{n+1}^{2}(x, y)=(\alpha-\beta)\left(\left(\alpha^{*}\right)^{2} \alpha^{2 n+1}-\left(\beta^{*}\right)^{2} \beta^{2 n+1}\right) .
$$

Proof. Using Binet formula and equation (2.5), we obtain

$$
\begin{aligned}
& y Q B F_{n}^{2}(x, y)+Q B F_{n+1}^{2}(x, y) \\
= & y\left(\frac{\alpha^{*} \alpha^{n}-\beta^{*} \beta^{n}}{\alpha-\beta}\right)^{2}+\left(\frac{\alpha^{*} \alpha^{n+1}-\beta^{*} \beta^{n+1}}{\alpha-\beta}\right)^{2} \\
= & \frac{1}{(\alpha-\beta)^{2}}\left\{\begin{array}{c}
y\left(\alpha^{*}\right)^{2} \alpha^{2 n}-y \beta^{*} \alpha^{*}-y \alpha^{*} \beta^{*}(\alpha \beta)^{n}+y\left(\beta^{*}\right)^{2} \beta^{2 n}+\left(\alpha^{*}\right)^{2} \alpha^{2 n+2} \\
-\beta^{*} \alpha^{*}(\alpha \beta)^{n+1}-\alpha^{*} \beta^{*}(\alpha \beta)^{n+1}+\left(\beta^{*}\right)^{2} \beta^{2 n+2}
\end{array}\right\} \\
= & \frac{1}{(\alpha-\beta)^{2}}\left\{y\left(\alpha^{*}\right)^{2} \alpha^{2 n}+\left(\alpha^{*}\right)^{2} \alpha^{2 n+2}+y\left(\beta^{*}\right)^{2} \beta^{2 n}+\left(\beta^{*}\right)^{2} \beta^{2 n+2}\right\} \\
= & \frac{\left(\alpha^{*}\right)^{2} \alpha^{2 n+1}-\left(\beta^{*}\right)^{2} \beta^{2 n+1}}{(\alpha-\beta)} .
\end{aligned}
$$

Other case can be done similarly.

Matrix method can use to get results for not only different identities but also algebraic representations in the study of recurrence relations.

$\operatorname{In}[10]$, the Pell quaternion matrix is defined by

$$
R(n)=\left(\begin{array}{cc}
R_{n} & R_{n-1} \\
R_{n-1} & R_{n-2}
\end{array}\right)
$$


and also was obtain equality as follow

$$
\left(\begin{array}{cc}
R_{n} & R_{n-1} \\
R_{n-1} & R_{n-2}
\end{array}\right)=\left(\begin{array}{ll}
R_{2} & R_{1} \\
R_{1} & R_{0}
\end{array}\right)\left(\begin{array}{ll}
2 & 1 \\
0 & 1
\end{array}\right)^{n-2}
$$

where $n \geq 2$ is an integer.

Now, we define the matrix for $Q B F_{n}(x, y)$ and $Q B L_{n}(x, y)$. The matrix $Q B F_{n}(x, y)(n)$ and $Q B L_{n}(x, y)(n)$ that play role of $R(n)$. These are

$$
Q B F_{n}(x, y)(n)=\left(\begin{array}{cc}
Q B F_{n+1}(x, y) & y Q B F_{n}(x, y) \\
Q B F_{n}(x, y) & y Q B F_{n}(x, y)
\end{array}\right)
$$

and

$$
Q B L_{n}(x, y)(n)=\left(\begin{array}{cc}
Q B L_{n+1}(x, y) & y Q B L_{n}(x, y) \\
Q B L_{n}(x, y) & y Q B L_{n}(x, y)
\end{array}\right)
$$

for $n \geq 1$.

Theorem 2.11. For an integer $n \geq 1$, we have

$$
Q B F_{n}(x, y)(n)=\left(\begin{array}{ll}
Q B F_{2}(x, y) & y Q B F_{1}(x, y) \\
Q B F_{1}(x, y) & y Q B F_{0}(x, y)
\end{array}\right)\left(\begin{array}{cc}
x & y \\
1 & 0
\end{array}\right)^{n-1}
$$

and

$$
Q B L_{n}(x, y)(n)=\left(\begin{array}{ll}
Q B L_{2}(x, y) & y Q B L_{1}(x, y) \\
Q B L_{1}(x, y) & y Q B L_{0}(x, y)
\end{array}\right)\left(\begin{array}{cc}
x & y \\
1 & 0
\end{array}\right)^{n-1}
$$

Proof. Induction method can be used to prove it. Let $n=1$, then basis step is clear. Now let us assume that the equation is valid for $n=k-1$. For $n=k$, it becomes

$$
\begin{aligned}
& \left(\begin{array}{ll}
Q B F_{2}(x, y) & y Q B F_{1}(x, y) \\
Q B F_{1}(x, y) & y Q B F_{0}(x, y)
\end{array}\right)\left(\begin{array}{ll}
x & y \\
1 & 0
\end{array}\right)^{k-1} \\
= & \left(\begin{array}{ll}
Q B F_{2}(x, y) & y Q B F_{1}(x, y) \\
Q B F_{1}(x, y) & y Q B F_{0}(x, y)
\end{array}\right)\left(\begin{array}{ll}
x & y \\
1 & 0
\end{array}\right)^{k-2}\left(\begin{array}{ll}
x & y \\
1 & 0
\end{array}\right) \\
= & \left(\begin{array}{cc}
Q B F_{k}(x, y) & y Q B F_{k-1}(x, y) \\
Q B F_{k-1}(x, y) & y Q B F_{k-2}(x, y)
\end{array}\right)\left(\begin{array}{ll}
x & y \\
1 & 0
\end{array}\right) \\
= & \left(\begin{array}{cc}
Q B F_{k+1}(x, y) & y Q B F_{k}(x, y) \\
Q B F_{k}(x, y) & y Q B F_{k-1}(x, y)
\end{array}\right) .
\end{aligned}
$$

which completes the proof. The other case can be done similarly. 


\section{Conclusion}

This work studied bivariate Fibonacci and Lucas quaternion polynomials. Since bivariate Fibonacci and Lucas quaternion polynomials were not intensive studied until now, we expect to find in the future more and surprising new properties. For this purpose, Fibonacci and Lucas quaternion polynomials was used and investigated in detail particularly in the first part. Also in the other part, Binet formulas, generating functions, matrix representation and some identities of bivariate Fibonacci and Lucas quaternion polynomials were computed. Quaternions have great importance as they are used in quantum physics, applied mathematics, graph theory and differential equations. Thus, in our future studies we plan to examine bivariate Fibonacci and Lucas octonion polynomials and their key features.

\section{REF E R E N C E S}

1. M. Catalani : Identities for Fibonacci and Lucas Polynomials derived from a book of Gould. /arxiv.org/abs/math/0407105.

2. P. Catarino: A note on h(x)-Fibonacci quaternion polynomials. Chaos, Solitons and Fractals. 77 (2015), 1-5.

3. S. HALiCI: On Fibonacci quaternions. Adv. Appl. Clifford Algebras. 22 (2012), 312-327.

4. A. İPEK: On $(p, q)$-Fibonacci quaternions and their Binet formulas, generating function and certain binomial sums. Adv. Appl. Clifford Algebras. 27 (2017), 1343-1351.

5. N. KILIC: The $h(x)$-Lucas quaternion polynomials. Annales Mathematicae et Informaticae. 47 (2017), 119-128.

6. E. G. Kocer and S. Tuncez: Bivariate Fibonacci and Lucas like polynomials. Gazi University Journal of Science. 29(1) (2016), 109-113.

7. A. Nalli and P. HaukKanen: On generalized Fibonacci and Lucas polynomials. Chaos, Solitons and Fractals. 42 (2009), 3179-3186.

8. A. Özkoç and A. Porsuk: A note for the (p;q)-Fibonacci and Lucas quaternions polynomials. Konuralp Journal of Mathematics. 5(2) (2017), 36-46.

9. A. ÖzKoÇ ÖzTürk and A. Porsuk: Some remarks regarding the ( $p ; q)$-Fibonacci and Lucas octonion polynomials. Universal Journal of Mathematics and Applications. 1(1) (2018), 46-53.

10. A. Szynal-Liana and I. Wloch: The Pell Quaternions and the Pell Octonions. Adv. App Clifford Algebras. 26 (2016), 435-440.

Arzu Özkoç Öztürk

Duzce University

Science and Art Faculty

Department of Mathematics

Duzce, Turkey 
arzuozkoc@duzce.edu.tr

Faruk Kaplan

Duzce University

Science and Art Faculty

Department of Mathematics

Duzce, Turkey

farkaplan@gmail.com 